Onkologe 2016 $22: 812-814$

DOI 10.1007/s00761-016-0117-2

(c) Springer-Verlag Berlin Heidelberg 2016

CrossMark

\section{J. Meran}

Interne Abteilung, Krankenhaus der Barmherzigen Brüder Wien, Wien, Österreich

\title{
Ethik in der Onkologie - Indikation und Respekt vor der Autonomie
}

Autonomie ist also der Grund der Würde der menschlichen und jeder vernünftigen Natur. (I. Kant, GMS: BA 79)

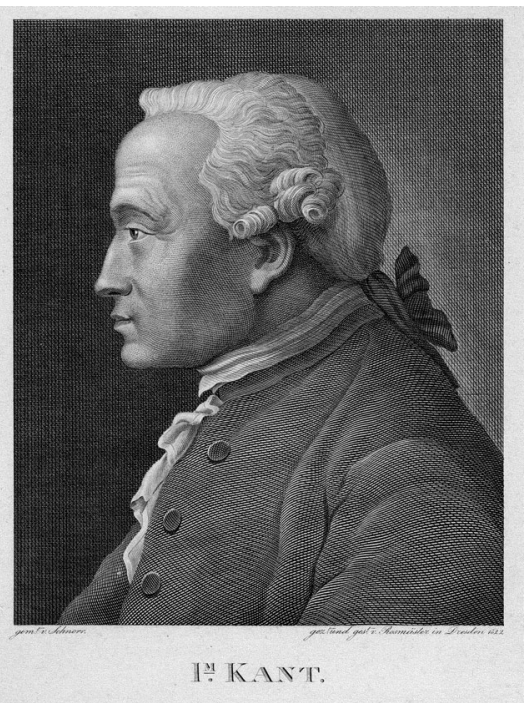

Das Leitthema „Ethik in der Onkologie “ beleuchtet weniger spektakuläre, dafür im ärztlichen Alltag umso wichtigere Grundfragen. Die Indikation ist dabei ein Schlüsselbegriff, dessen normativ-wertende Substanz klar herausgestellt wird und einen roten Faden in den folgenden Beiträgen zu dialogischen Konkretisierungen ethischer Aspekte bildet. Personalisierung und Priorisierung sind zwar neue Akzente in der Onkologie, kommen aber ohne die alte Grundfrage der Indikation nicht aus. Da Indikation weit mehr bedeutet als Leitlinien $\mathrm{zu}$ folgen und neben der ständigen Herausforderung fachlicher Aktualität untrennbar mit den persönlichen Therapiezielen zusammenhängt, greift eine abstrakte, vom Dialog mit den Patienten abgekoppelte Indikationsstellung zu kurz. Der Dialog über das Therapieziel ist quasi voraussetzend für das Finden der richtigen Indikation, zu der dann die endgültige Zustimmung (,informed consent") noch erfolgen muss.

Gerade am Ende des Lebensbogens drängen sich die Fragen dichter und die onkologische Ärzte sind bei der ständigen Suche nach dem aktuellen Therapieziel herausgefordert, da sich das Ziel dynamisch mit der medizinischen Situation ebenso wie mit den gegebenen therapeutischen Möglichkeiten verändern kann und soll. Wie ein Kompass kann der Patientenwille dabei Orientierung bieten - und der Respekt vor diesem Willen begründet Würde auch in schwierigsten Lebenslagen, wie schon Immanuel Kant gelehrt hat. Erst die Kombination von informierter Zustimmung als Ausdruck der Autonomie und die fachlich geprüfte Indikation rechtfertigt therapeutisches Handeln. Die autonome Zustimmung ist damit die Bedingung der Möglichkeit, ärztliche Behandlungsvorschläge umsetzen zu können.

Das strukturierte Ethikkonsil kann gute Hilfen bieten, um verflochtene und kontroverse Fragen sowie unterschiedliche Perspektiven $\mathrm{zu}$ ordnen und im Versuch, den Willen der Patienten zu ermitteln, voranzukommen. Die Handlungsverantwortung bleibt bei den behandelnden Ärzten. Urteilskraft forderte Immanuel Kant für moralische Entscheidungen, und die gebildete Urteilskraft des Arztes ist in der kritischen Reflexion medizinischer Entscheidungen gefragt. Die Begründung dieser Entscheidungen ist das ethische Element des ärztlichen Handelns. Wenn Grenzsituationen in der Onkologie Abwägungen und Wer- tungen erfordern, hilft eine Struktur, die Transparenz gewährleistet und Prinzipien vor dem Hintergrund medizinischer Fachexpertise verdeutlicht. Ein wesentliches Element bei dieser Form eines qualifizierten und ethisch informierten Konfliktmanagements ist dabei das Finden und pragmatische Bewerten der möglichen Handlungsoptionen, wobei es sich um eine Prozessberatung im Sinne einer strukturierten Moderation handelt.

Auch die ethische Analyse lebensverlängernder Behandlungen rekurriert auf die Bedeutung der Kommunikation und möglichst vorausschauender Planung wie das Advance Care Planning, um den Wünschen und Präferenzen der Patienten gerecht zu werden. Entscheidungen zur Begrenzung lebensverlängernder Behandlung sollten vor dem Hintergrund der sich im Krankheitsverlaufändernden Therapieziele am Lebensende getroffen werden. Idealerweise handelt es sich um eine gemeinsame Entscheidungsfindung („shared decision making“), die der Autonomie der Patienten entsprechenden Raum auch in den Grenzbereichen am Lebensende gibt.

Eine solche Grenzentscheidung betrifft die palliative Sedierung, die als Anwendungsbeispiel besonders deutlich werden lässt, wie wichtig die Rechtfertigung der Indikation und die dialogische Definition der Therapieziele ist. Dabei kommt dem Prinzip der Proportionalität in dem Sinne besondere Bedeutung zu, dass genau soviel Sedierung angemessen ist, wie zur Erreichung des Ziels der Symptomlinderung notwendig ist. Die intermittierende, und mehr noch die permanente, medikamentöse Redukti- 
Hier steht eine Anzeige.

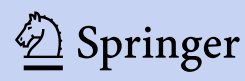


on des menschlichen Wachbewusstseins erscheint in besonderem Maße rechtfertigungsbedürftig.

Wieviel auch an psychologischer Herausforderung in der Betreuung onkologischer Patienten steckt, soll durch den Beitrag zu den Bewältigungsstrategien gezeigt werden. Im komplexen System der Krankheitsverarbeitung können auch neue Ressourcen entdeckt und verstärkt werden und im Rahmen einer begrenzten Autonomie die eigenen Kräfte trotz widriger Umstände wachsen. Der Mensch selbst bleibt das Maß und der Zweck an sich, an dem sich jegliche Therapie orientieren soll.

Nun sage ich: Der Mensch, und überhaupt jedes vernünftige Wesen, existiert als Zweck an sich selbst, nicht bloß als Mittel zum beliebigen Gebrauche für diesen oder jenen Willen, sondern muss in allen seinen, sowohl auf sich selbst als auch auf andere Vernünftige Wesen gerichteten Handlungen, jederzeit zugleich als Zweck betrachtet werden. (I. Kant GMS, BA 64)

Für die Schriftleiter

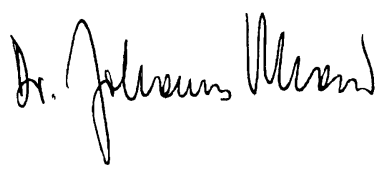

Johannes Meran

Für die Herausgeber

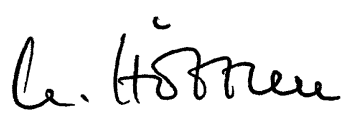

Klaus Höffken

\section{Korrespondenzadresse}

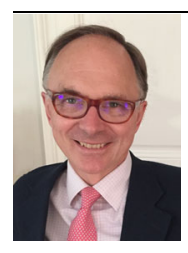

Prim. Univ. Prof. Dr.

\section{J. Meran, M.A.}

Interne Abteilung,

Krankenhaus der

Barmherzigen Brüder Wien Johannes von Gott Platz 1, 1020 Wien, Österreich johannes@meran-online.at

Interessenkonflikt. J. Meran gibt an, dass kein Interessenkonflikt besteht.

\section{ONKO-Internetportal}

Aktuelles von den Kongressen ESMO und DGHO

Im Herbst ist die Kongressdichte für Onkologen und onkologisch tätige Ärzte besonders hoch. Alljährlich geben Tagungen der deutschen und europäischen Fachgesellschaften - etwa der European Society for Medical Oncology (ESMO) oder der Deutschen Gesellschaft für Hämatologie und Medizinische Onkologie (DGHO) - einen umfassenden Überblick über den aktuellen Stand der Krebsforschung. Das Redaktionsteam des ONKO-Internetportals war vor Ort und liefert Expertenmeinungen zu den wichtigsten Themen aus erster Hand.

Einen Überblick über das Kongressgeschehen des ESMO 2016 in Kopenhagen können sich Ärzte online unter www.krebsgesellschaft.de/esmo_2016 verschaffen. Mehr als 20.000 Teilnehmerinnen und Teilnehmer diskutierten dort die Ergebnisse aktueller Krebsstudien. Im Mittelpunkt standen neben Indikationen wie Brust- und Lungenkrebs auch gastrointestinale Tumoren sowie seltenere Krebsarten. Schwerpunkte im Bereich der Krebstherapie waren die Immuntherapie, zielgerichtete Therapien und Aspekte der personalisierten Medizin.

Aktuelle Impressionen von der DGHO-Jahrestagung 2016 liefert das ONKO-Internetportal ab sofort mit Video-Interviews unter www.krebsgesellschaft.de/dgho_2016.

Themenschwerpunkte auf dem DGHO in Leipzig waren neben zahlreichen neuen Therapieansätzen und der Interdisziplinarität bei der Versorgung von Krebspatienten die Liquid Biopsy und molekulare Diagnostik.

Quelle

Deutsche Krebsgesellschaft e.V. (DKG)

www.krebsgesellschaft.de

\section{Helmut-Wölte-Preis für Psychoonkologie 2017}

Bonn. Die Helmut-Wölte-Stiftung schreibt den Helmut-Wölte-Preis für Psychoonkologie für das Jahr 2017 aus. Der Preis ist mit 2.500 Euro dotiert. Die Bewerbungsunterlagen sind unter www.helmut-woelte-stiftung.de downloadbar.

Die Stiftung unterstützt durch den Preis Initiativen zur psychosozialen Versorgung von Krebspatienten und ihren Angehörigen, die bei wissenschaftlich fundierter Praxis innovativen, zukunftsweisenden Modellcharakter besitzen. Es werden nur Projekte berücksichtigt, die sich bereits in der Phase ihrer Realisierung befinden.

Der Preisträger wird durch eine Jury bestehend aus Stiftungsvorstand und beratenden Wissenschaftlern ermittelt.

Bewerbungen aus dem deutschsprachigen Raum und Anfragen sind in digitaler Form unter dem Stichwort "Helmut-Wölte-Preis 2017" zu richten an:

PD Dr. Andrea Schumacher Medizinische Klinik A, Universitätsklinikum Münster, 48129 Münster

E-Mail andrea.schumacher@

helmut-woelte-stiftung.de

Einsendeschluss ist der 15. März 2017. 